\title{
HES1 promotes breast cancer stem cells by elevating Slug in triple-negative breast cancer
}

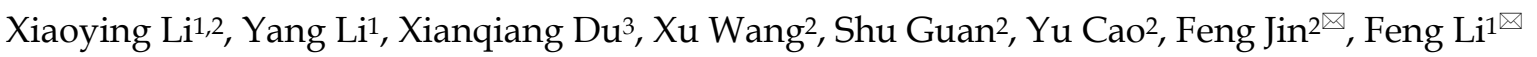 \\ 1. Department of Cell Biology, Key Laboratory of Cell Biology, National Health Commission of the PRC, and Key Laboratory of Medical Cell Biology, Ministry \\ of Education of the PRC, China Medical University, No. 77, Puhe Road, Shenyang North New Area, 110122 Shenyang, Liaoning, China. \\ 2. Department of Breast Surgery, The First Affiliated Hospital of China Medical University, 155 Nanjing Road, 110001 Shenyang, China. \\ 3. Department of Breast Surgery, Affiliated Quanzhou First Hospital of Fujian Medical University, Anji Road, Quanzhou, China.
}

$\triangle$ Corresponding authors: Feng Li (E-mail: lifeng@cmu.edu.cn); Feng Jin (E-mail: jinfeng@cmu.edu.cn).

(C) The author(s). This is an open access article distributed under the terms of the Creative Commons Attribution License (https://creativecommons.org/licenses/by/4.0/). See http://ivyspring.com/terms for full terms and conditions.

Received: 2020.09.20; Accepted: 2020.10.21; Published: 2021.01.01

\begin{abstract}
Triple-negative breast cancer (TNBC) is the most aggressive subtype of breast cancer. TNBC is enriched with breast cancer stem cells (BCSCs), which are responsible for cancer initiation, cancer progression and worse prognosis. Our previous study found that HESI was overexpressed and promoted invasion in TNBC. However, the role of HESI in modulating BCSC stemness of TNBC remains unclear. Here, we found that HESI upregulates Slug both in transcriptional level and in protein level. HES1 also has a positive correlation with Slug expression in 150 TNBC patient samples. TNBC patients with high HESI and Slug levels show worse prognosis in both progression-free survival and overall survival analyses. Survival analyses indicate that the effects of HESI on survival prognosis may depend on Slug. Furthermore, we reveal that HESI is a novel transcriptional activator for Slug through acting directly on its promoter. Meanwhile, HES1 knockdown reduces BCSC self-renewal, BCSC population, and cancer cell proliferation in TNBC, whereas overexpression of Slug restores the oncogenic function of HES1, both in vitro and in vivo, suggesting that HESI performs its oncogenic role through upregulating Slug. Taken together, HES1 promotes BCSC stemness properties via targeting Slug, highlighting that HESI might be a novel candidate for BCSC stemness regulation in TNBC and providing new clues for identifying promising prognostic biomarkers and therapeutic targets of TNBC.
\end{abstract}

Key words: stem cells, breast cancer

\section{Introduction}

Breast cancer is the most commonly diagnosed malignancy and the leading cause of cancerassociated mortality among women worldwide [1,2]. Triple-negative breast cancer (ER-, PR-, and HER2-negative, TNBC), the most aggressive subtype, accounts for approximately $10-24 \%$ of all breast cancers and is associated with advanced stage, high recurrence rate, frequent distant metastasis, chemoresistance and worse prognosis [3]. Due to the lack of effective targeted therapy for TNBC, striving for novel biomarkers and specific targets is regarded to be imperative to both theoretical studies and clinical practice.

Recently, more and more research has indicated that TNBC is enriched with cancer stem cells (CSCs), which resulted in remarkably heterogeneous [4].
Cancer stem cells (CSCs) represent a dynamic small subpopulation of cancer cells, characterized by self-renewal, pluripotency and limitless proliferative properties [5]. Breast cancer stem cells (BCSCs) are considered as the source of cancer aggression and metastasis, poor prognosis, drug resistance and early recurrence in TNBC [6]. Therefore, it is essential to identify key stemness regulators and BCSC-related pathways, which can be applied to predict cancer aggressive potentiality and acted as molecular specific targets for the treatment of TNBC.

Hairy and enhancer of split homolog-1 (HES1) is a basic helix-loop-helix (bHLH) transcriptional factor that plays a critical role in many physiological processes and various morphogenetic events, especially gaining and retaining CSC stemness 
capacity, inhibiting cell committed differentiation [7, 8]. HES1 exerts its dual functions of transcriptional activation and repression [9]. Recent many studies showed that HES1 overexpression resulted in chemoresistance in lung cancers, intestine cancers, and pancreatic cancers owing to elevating stemness properties acquisition $[8,10,11]$. Our previous study also found that HES1 is highly expressed in TNBC than in other subtypes of breast cancer and significantly correlated with advanced TNM stage and poor prognosis. In addition, HES1 overexpression induced epithelial to mesenchymal transition (EMT) process and promoted cancer invasion in TNBC [12]. However, it has not been clarified whether HES1 promotes TNBC to gain BCSC phenotype and regulates BCSC stemness properties.

It is well known that EMT is the process characterized by epithelial cancer cells acquiring a mesenchymal gene program which facilitates migration and invasion [13]. It has been demonstrated that some transcription factors (such as Twist, Snail, Slug, ZEB1, ZEB2) involved in EMT process, which were counted as EMT-inducing transcription factors (EMT-TFs) [14, 15]. Over the past few years, it has been demonstrated that EMT is closely associated with CSC stemness properties [16]. In breast cancer, interconversion of CSC and non-CSC might be a relatively common phenomenon that is driven by EMT process [17]. Based on the above, it led us to hypothesize that HES1 might be involved in acquisition and maintenance of BCSC stemness properties in TNBC.

In the present study, we investigated the regulatory role of HES1 in EMT-TFs and BCSC stemness in TNBC. First, we found that HES1 could act directly on Slug promoter to activate its transcription, which is a major EMT-TF. Moreover, there were the positively significant correlation between HES1 expression and Slug expression in 150 TNBC patient specimens. Furthermore, HES1 functioned as an important contributor to regulate BCSC stemness and cancer cell proliferation in TNBC through elevating Slug expression, both in vitro and in vivo. This study might provide theoretical basis for finding novel prognostic markers and therapeutic targets of TNBC.

\section{Materials and Methods}

\section{Patient specimens}

All patients were diagnosed with triple-negative invasive ductal carcinoma in the First Affiliated Hospital of China Medical University. None of the patients had received preoperational radiotherapy or chemotherapy. Breast cancer specimens $(n=150$ patients) were obtained from patients hospitalized between September 2010 and September 2011. The deadline date of follow-up was September 2020. All 150 patients had a definite histological pathological diagnosis of breast cancer according to the American Joint Committee Cancer (AJCC) standard. The average age of the 150 patients was 47 years. This study was approved by Ethics Committee of China Medical University (Approval number: AF-SOP-071.1-01) with written, informed consent was obtained from each participating patient.

\section{Immunohistochemistry (IHC)}

IHC was performed as previously described [12]. Briefly, sections from paraffin-embedded tumor tissues from patients underwent surgical dissections or transplanted nude mice were incubated with primary antibodies. Results were evaluated by two pathologists who were blinded to the experiment separately. Slug immunoreactivity was quantified using a combined "H score", which assesses both the staining intensity and (0, negative; 1 , weak; 2 , moderate; 3 , strong) and the percentage of cells positively stained $(0,<5 \% ; 1,5-25 \% ; 2,26-50 \% ; 3$, $51-75 \% ; 4,76-100 \%)$. HES1 scores were estimated according to previously described [12]. Scores of more than or equal to 4 were defined as positive expression.

\section{Cell culture and transfection}

Triple-negative breast cancer cell lines MDAMB-231 and HCC-1937 were obtained from ATCC (Manassas, VA, USA). Cell lines were cultured in DMEM (Gibco) with 10\% FBS (Gibco). MDA-MB-231 CSCs and HCC1937 CSCs were induced and cultured in DMEM-F12 (Gibco, Thermo Fisher Scientific), containing 2\% B27 (Gibco), b-FGF $10 \mu \mathrm{g} / \mathrm{L}$ (Promega), EGF $20 \mu \mathrm{g} / \mathrm{L}$ (Promega), as previously described. All cells were incubated in a $5 \% \mathrm{CO} 2$ air at $37{ }^{\circ} \mathrm{C}$. The shRNA expressing lentivirus for HES1 and the Slug-overexpression lentivirus were purchased from Hanheng Company (Wuhan, China) and GeneChem Company (Shanghai, China). The sequences of HES1shRNA used to knockdown HES1 were GGACATTCT GGAAATGACA. Puromycin $(10 \mathrm{mg} / \mathrm{ml}$; Sigma, USA) was used to select stably transfected cells. Human Slug cDNA was amplified by PCR and cloned into pcDNA3.1 plasmid. Triple-negative cancer cells were transfected with Slug plasmid by Lipofactamine 3000 (Invitrogen, Carlsbad, CA, USA).

\section{Western blot}

In brief, cells were lysed with RIPA buffer containing $1 \%$ protease inhibitor cocktail (Roche, Germany) on ice for $30 \mathrm{~min}$ and then lysates were centrifuged. Protein concentrations were measured using the BCA assay kit (KeyGen). Cell lysates were 
separated by SDS-PAGE and transferred to polyvinylidene fluoride membranes (Millipore, Billerica, MA, USA) and the membranes were incubated with primary antibodies overnight at $4{ }^{\circ} \mathrm{C}$. The primary antibodies used in western blot were: anti-HES1 (1:500, ab71559, Abcam), anti-Twist (1:1000, 46702, CST), anti-Snail (1:1000, 3829, CST), anti-Slug (1:1000, 9585, CST), anti-ZEB1 (1:1000, 3396 , CST), anti-ZEB2 (1:1000, 97885, CST), anti-SOX2 (1:1000, ab92494, Abcam), anti-OCT4 (1:1000, 2750, CST), anti-Nanog (1:1000, ab109250, Abcam) and anti- $\beta$-actin (1:1000, ab8227, Abcam). All western blots were derived from the same experiment and were processed in parallel.

\section{RNA isolation and quantitative real-time PCR (qRT-PCR)}

Total RNA was extracted from cells using Trizol reagent (Invitrogen, Carlsbad, USA). Quantitative real-time PCR was performed using the SYBR Green mix (Applied Biosystems) on a 7500 Fast Real-Time PCR System (Applied Biosystems). The sequences of the primers were as follows (5'-3'): HES1 (TCAACAC GACACCGGATAAAC and GCCGCGAGCTATCTTT CTTCA); Twist (GTCCGCAGTCTTACGAGGAG and GCTTGAGGGTCTGAATCTTGCT); Snail (TCGGAA GCCTAACTACAGCGA and AGATGAGCATTGGC AGCGAG); Slug (CGAACTGGACACACATACA GTG and CTGAGGATCTCTGGTTGTGGT); ZEB1 (GATGATGAATGCGAGTCAGATGC and ACAGCA GTGTCTTGTTGTTGT); ZEB2 (CAAGAGGCGCAAA CAAGCC and GGTTGGCAATACCGTCATCC) and GAPDH (GAGTCAACGGATTTGGTCGTATTG and CCTGGAAGATGGTGATGGGATT). The fold change of gene expression levels was quantified using the $2-\Delta \Delta \mathrm{Ct}$ method. GAPDH was amplified as endogenous control.

\section{ChIP (Chromatin immunoprecipitation) assay}

Potential transcription factor HES1-binding sequences within Slug promoter region were identified by JASPAR database. According to the protocol of ChIP assay kit (Merk Millipore); MDAMB-231 cells were cross-linked with $1 \%$ formaldehyde at $37{ }^{\circ} \mathrm{C}$ for $10 \mathrm{~min}$ after washing. Cells were resuspended in lysis buffer, sonicated to shear DNA and sonicates were immunoprecipitated with antiHES1 antibody (ab70576, Abcam) or negative control IgG at $4{ }^{\circ} \mathrm{C}$ overnight. The immunocomplex was collected on protein A/G-agarose and washed in turn with low salt, high salt, lithium chloride buffer and TE buffer. After elution and reverse cross-linking, the DNA was extracted and analyzed by PCR using primer pairs for the Slug promoter: Slug (BE1) forward: 5'-CAAGGAGGACTCCTGCTCTC-3', reverse: 5'-CCTCTGGCTTTTACTCCAGG-3'; Slug
(BE2) forward: 5'-GCTAACACGGTGACATGAGT-3', reverse: 5'-CACGCAAGGCTGCAGT-3'; Slug (BE3) forward: 5'- CССТCCTAGCTCCCAGA-3', reverse: 5'-CСТCTCCACTGAAATCTCAA-3'.

\section{Luciferase dual reporter assays}

The promoter sequence of Slug gene (from -2000 to -1) was obtained from the UCSC website. Partial sequences of the promoter were amplified by PCR, and the fragments were cloned into the pGL3-basic vector. MDA-MB-231 cells were co-transfected with pGL3-basic or pGL3-Slug-Luc together with sh-HES1 or sh-NC, and pRL-TK plasmid. After $24 \mathrm{~h}$, the cells were lysed and luciferase activities were analyzed using a Promega dual-luciferase assay kit according to the manufacturer's instructions.

\section{Flow cytometry assay}

All cell lines were digested with $0.25 \%$ trypsin and washed with PBS three times. Then cells were resuspended in $100 \mu \mathrm{L}$ PBS and incubated with anti-CD44-APC $(1.25 \mu \mathrm{l} /$ test) and anti-CD24-PE (5 $\mu \mathrm{l} /$ test) (Biolegend, San Diego, USA), or with their controls at $4{ }^{\circ} \mathrm{C}$ for $30 \mathrm{~min}$. After incubation, the cell distribution was measured using a MACSQuantTM Flow Cytometer (Miltenyi Biotec).

\section{Sphere-formation assay and colony formation assay}

Sphere-formation assay was performed as previously described $[18,19]$. Essentially, MDA-MB231 and HCC-1937 cell suspension $\left(1 \times 10^{3} /\right.$ well $)$ were plated in ultralow adhesion plates (Corning, Kraemer, CA). The cells grown in $2 \mathrm{ml}$ serum-free DMEM-F12 with $10 \mu \mathrm{g} / \mathrm{L}$ bFGF, $20 \mu \mathrm{g} / \mathrm{L}$ EGF and 2\% B27. For Colony formation assay, $2 \times 10^{3}$ MDA-MB-231 and HCC-1937 cells were seeded into $6 \mathrm{~cm}$ petri dishes and cultured at $37{ }^{\circ} \mathrm{C}$ in $5 \% \mathrm{CO} 2$ for 14 days. Then, cells were washed with PBS, fixed with $4 \%$ paraformaldehyde and stained with $0.5 \%$ crystal violet for number counting.

\section{Xenograft model}

Nude female mice ( 8 weeks-old) were used for studying tumorigenesis ability. Tumorsphese cells with limiting dilution $\left(1.0 \times 10^{3} ; 1.0 \times 10^{4} ; 1.0 \times 10^{5}\right.$ cells per mouse) were injected subcutaneously into second mammary fat pad. We adopted the method of tumorsphere isolation and cells count in our study, which was described in the previous researches in detail $[19,20]$. In essential, spheres were collected, enzymatically dissociated into single cells by $0.05 \%$ trypsin-EDTA solution for $2-3 \mathrm{~min}$ at $37{ }^{\circ} \mathrm{C}$. Disaggregate the tumorspheres using a $25 \mathrm{G}$ needle and syringe until a single cell suspension is produced. Single cells were counted by normal cell-counting 
method or using cell counter (Invitrogen Countess II Automated Cell Counter). The mice were randomized into the following three groups: MDA-MB-231/shNC, MDA-MB-231/sh-HES1 and MDA-MB-231/shHES1+oe-Slug. The tumor diameter and weights were measured on every 3 days. The tumor volume was calculated by width ${ }^{2} \times$ length $/ 2$. All mice were bred at pathogen-free conditions in the Animal Center. All animal experiments were approved by the Institutional Animal Care and Use Committee of China Medical University (Approval number: 2017007 M).

\section{Statistical analysis}

Statistical analyses were conducted using SPSS 20.0 (Chicago, IL, USA) and GraphPad Prism 8.0 software. All data are presented as the means \pm standard deviations (SD) and are representative of at least three experiments. Two-sided Student's $t$-test was performed between two groups. The correlation between HES1 and Slug expression was examined by Spearman's rank correlation test. $P<0.05, P<0.01$, $P<0.001$ and $P<0.0001$ were considered statistically significant.

\section{Results}

\section{HESI upregulates Slug and positively correlates with Slug expression in TNBC}

Our previous study showed that HES1 promotes the EMT process of TNBC cells [12]. To further explore how HES1 participates in the activation of EMT process, we examined the expression correlations

A
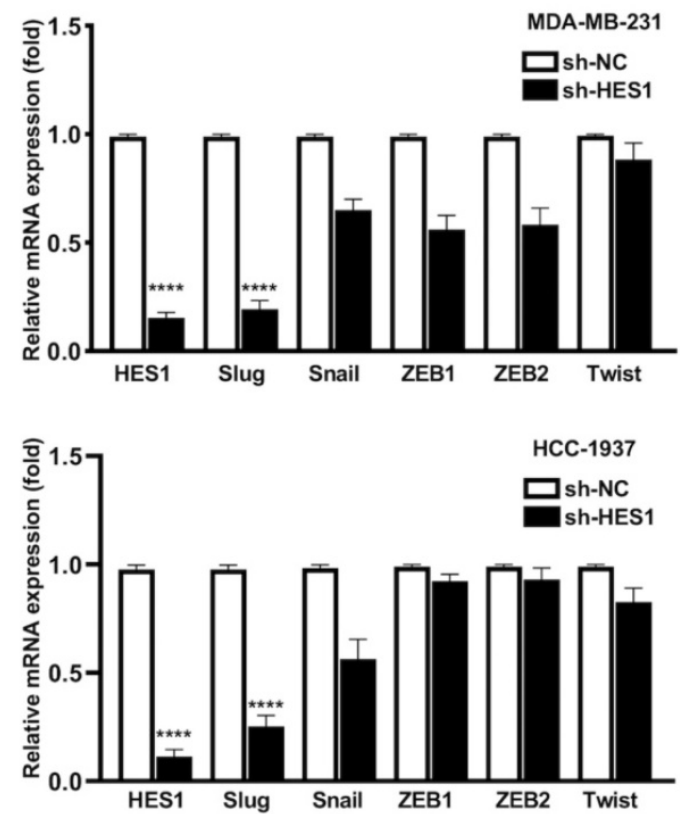

among HES1 and other EMT-TFs. In TNBC cell lines (MDA-MB-231 and HCC-1937), the mRNA and protein levels of Slug were the most significantly downregulated with loss of HES1 compared with other EMT-TFs (Snail, ZEB1, ZEB2, Twist) (Figure 1). Meanwhile, Slug was high endogenous level in TNBC cell lines, whereas other EMT-TFs (Snail, ZEB1, ZEB2 and Twist) were low endogenous level and slightly expressed in TNBC cell lines (Figure 1). Thus, these data suggested that Slug may serve as a major trigger of EMT in TNBC and HES1 contributes to EMT induction mainly through Slug. Subsequently, in our collected TNBC patient specimens $(n=150)$, there was a significantly positive correlation between HES1 and Slug expression (Figure 2).

To further evaluate the role of HES1 and Slug in TNBC development, we analyzed the relationship between HES1 and Slug expression and the clinical pathological factors. There was a significant correlation of HES1 or Slug expression with tumor size $(P=0.0262 ; P=0.0092)$, lymph node metastasis $(P=0.0129 ; \quad P=0.0060)$ and advanced TNM stage $(P=0.0001 ; P=0.0005)$ (Table 1$)$. To investigate the prognostic role of HES1 and Slug expression in TNBC, we performed Kaplan-Meier survival analysis. TNBC patients with HES1-positive had a significantly lower progression-free survival (PFS) $(P=0.019)$ and overall survival (OS) $(P=0.018)$ than $\mathrm{TNBC}$ patients with HES1-negative. There were the consistent trends in TNBC patients with Slug-positive (PFS: $P=0.003$; OS: $P=0.001$ ) compared with TNBC patients with Slug-negative (Figure 3A-D). TNBC patients with both HES1- and Slug- positive significantly correlated

B

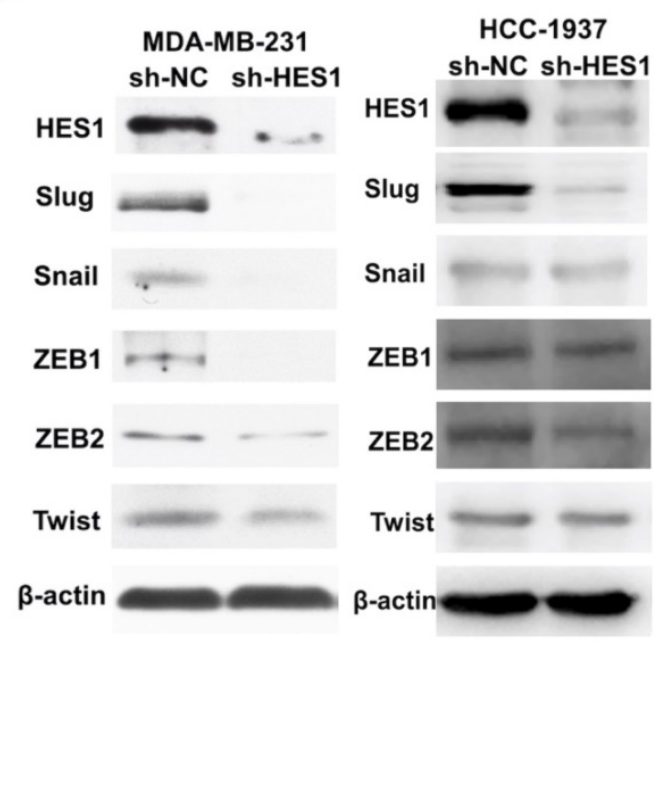

Figure 1. HES1 upregulates Slug in TNBC. A) Protein and B) mRNA levels of EMT-inducing transcription factors (Twist, Snail, Slug, ZEB1, ZEB2) was detected in HES1-knockdown TNBC cell lines (MDA-MB-231 and HCC-1937). 
with worse prognosis (PFS: $P=0.002$; OS: $P=0.001$ ) compared with TNBC patients with both HES1- and Slug- negative (Figure 3E-F). In addition, we also analyzed whether Slug level affects survival of TNBC patients in the context of HES1 negative and whether HES1 level has an impact on survival of TNBC patients under the circumstance of Slug negative. As shown in Figure 3G-H, there was a significant relationship between Slug expression and PFS $(P=0.046)$ in HES1-negative TNBC patients, as well as there is a remarkable correlation of high Slug level and lower OS $(P=0.022)$ in HES1-negative TNBC patients, implying that HES1 may affect survival prognosis dependent on Slug. Meanwhile, as shown in Figure 3I-J, there was no significant association of HES1 expression with PFS $(P=0.325)$ or OS $(P=0.309)$ in Slug-negative TNBC patients, further indicating that Slug might be a downstream gene of HES1. Thus, the above data suggest that HES1 upregulates Slug in
TNBC cells and HES1 level is correlated with Slug level in TNBC specimens. Moreover, HES1 might be an important progressive and prognostic factor in TNBC.

Table 1. Association of HES1 and Slug expression with the clinical pathological characteristics in TNBC

\begin{tabular}{|c|c|c|c|c|c|c|c|}
\hline \multirow{2}{*}{$\begin{array}{l}\text { Factors } \\
\text { Age }\end{array}$} & \multirow[t]{2}{*}{ Number } & \multicolumn{2}{|c|}{ HES1 expression } & \multicolumn{4}{|c|}{ Slug expression } \\
\hline & & Positive & Negative & $P$-value & Positive & Negative & $P$-value \\
\hline$\leq 47$ & 69 & 40 & 29 & 0.7539 & 42 & 27 & 0.7923 \\
\hline$>47$ & 81 & 49 & 32 & & 51 & 30 & \\
\hline \multicolumn{8}{|c|}{ Tumor size $(\mathrm{cm})$} \\
\hline$\leq 3$ & 77 & 39 & 38 & $0.0262^{*}$ & 40 & 37 & $0.0092^{*}$ \\
\hline$>3$ & 73 & 50 & 23 & & 53 & 20 & \\
\hline \multicolumn{8}{|c|}{ LN metastasis } \\
\hline Negative & 63 & 30 & 33 & $0.0129^{*}$ & 31 & 32 & $0.0060^{*}$ \\
\hline Positive & 87 & 59 & 28 & & 62 & 25 & \\
\hline \multicolumn{8}{|c|}{ TNM stage } \\
\hline I & 37 & 12 & 25 & $0.0001^{*}$ & 14 & 23 & $0.0005^{*}$ \\
\hline II-III & 113 & 77 & 36 & & 79 & 34 & \\
\hline
\end{tabular}

${ }^{*}$ Indicated statistical significance $(P<0.05)$.

A

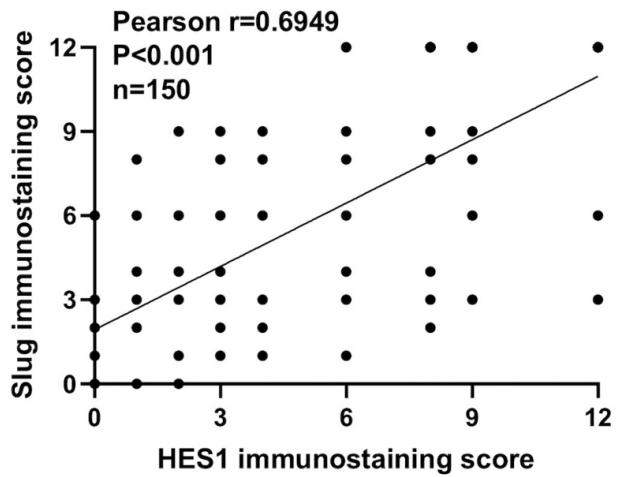

B

\section{HES1}
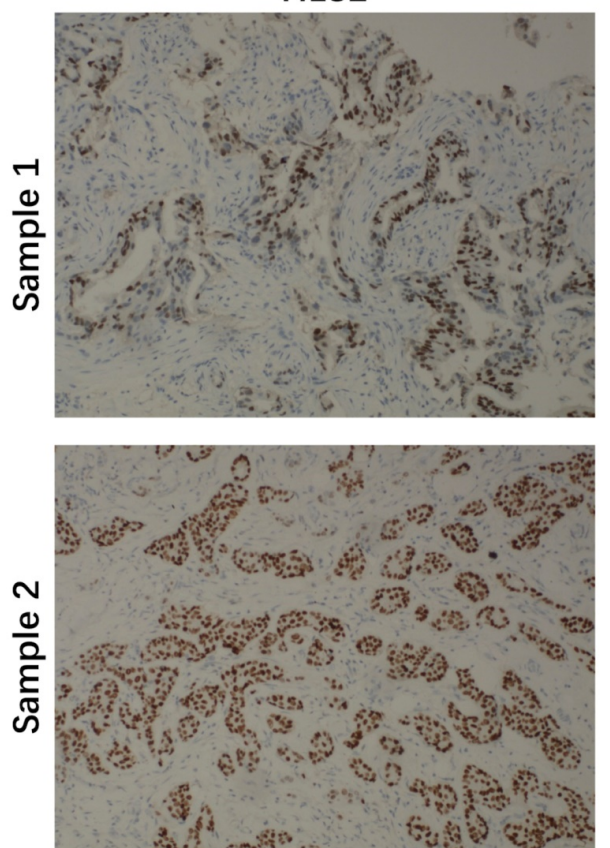

Slug
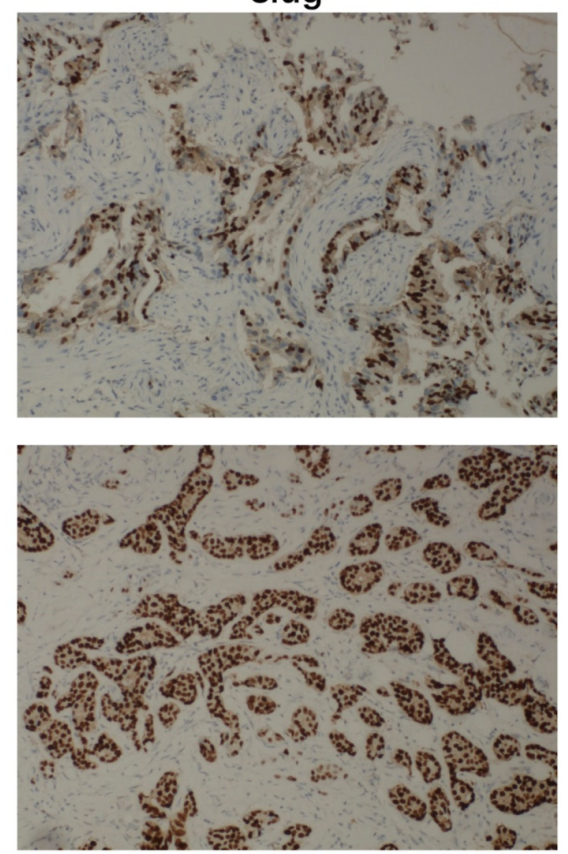

Figure 2. Correlation analysis of HESI and Slug in TNBC patients by IHC. A) The positive correlation between HES1 and Slug expression by IHC scores using Pearson's correlation analysis. B) Representative IHC staining with HES1 and Slug in TNBC tissues. 
A

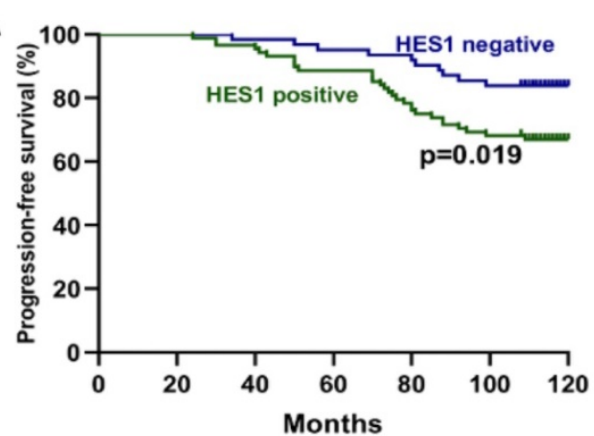

C

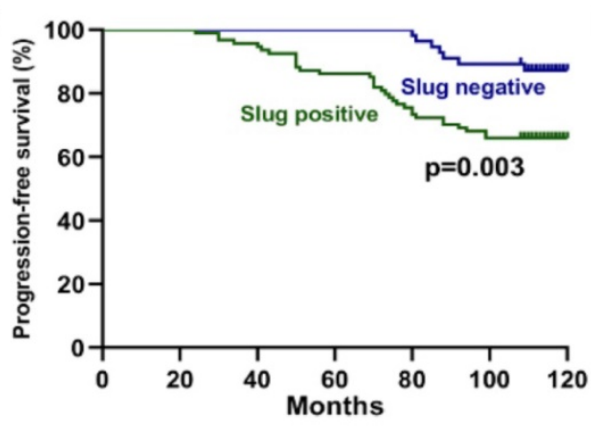

E

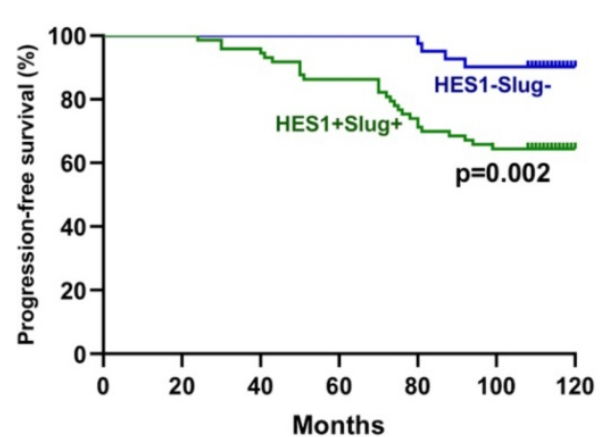

G

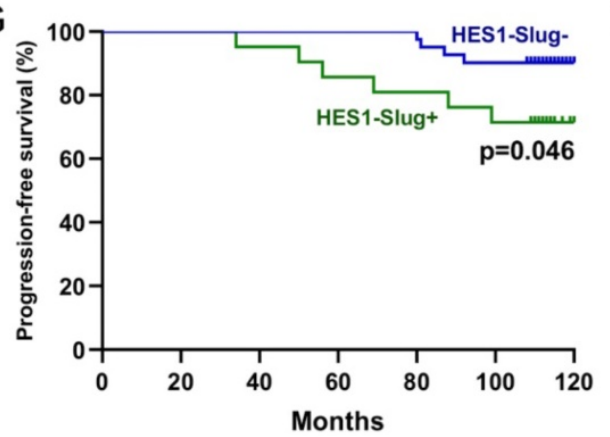

I

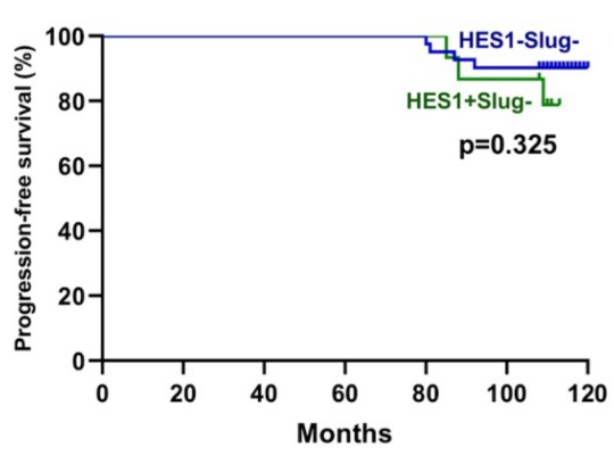

B
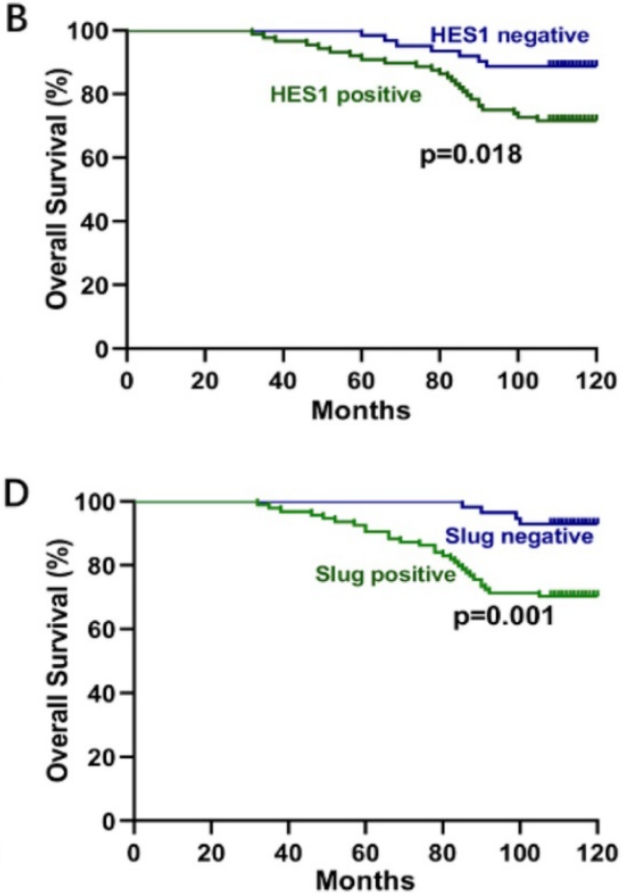

F
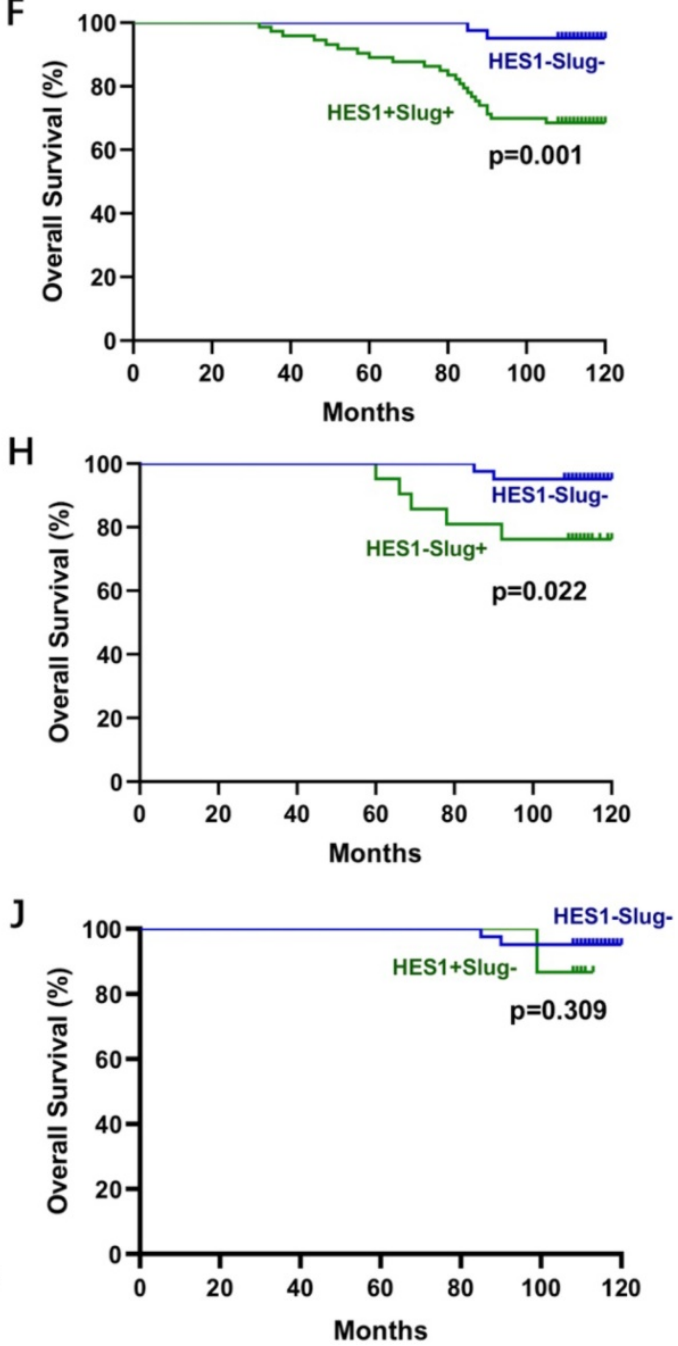

Figure 3. Correlation analysis between HES1 or Slug expression and survival in TNBC. A-B) The correlation of HES1 expression with PFS and B) OS of TNBC patients by Kaplan-Meier survival analysis. C-D) The correlation of Slug expression with PFS and OS of TNBC patients by Kaplan-Meier survival analysis. E-J) The correlation of both HESI and Slug expression with PFS and OS of TNBC patients by Kaplan-Meier survival analysis. 
A

Slug (NM_003068.4) chr8: $48917677-48921440$ (reverse complement)

Slug promoter region chr8: $48921440-48923440$ (reverse complement)

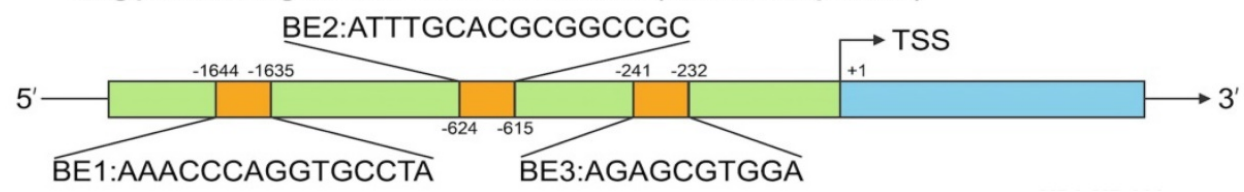

B
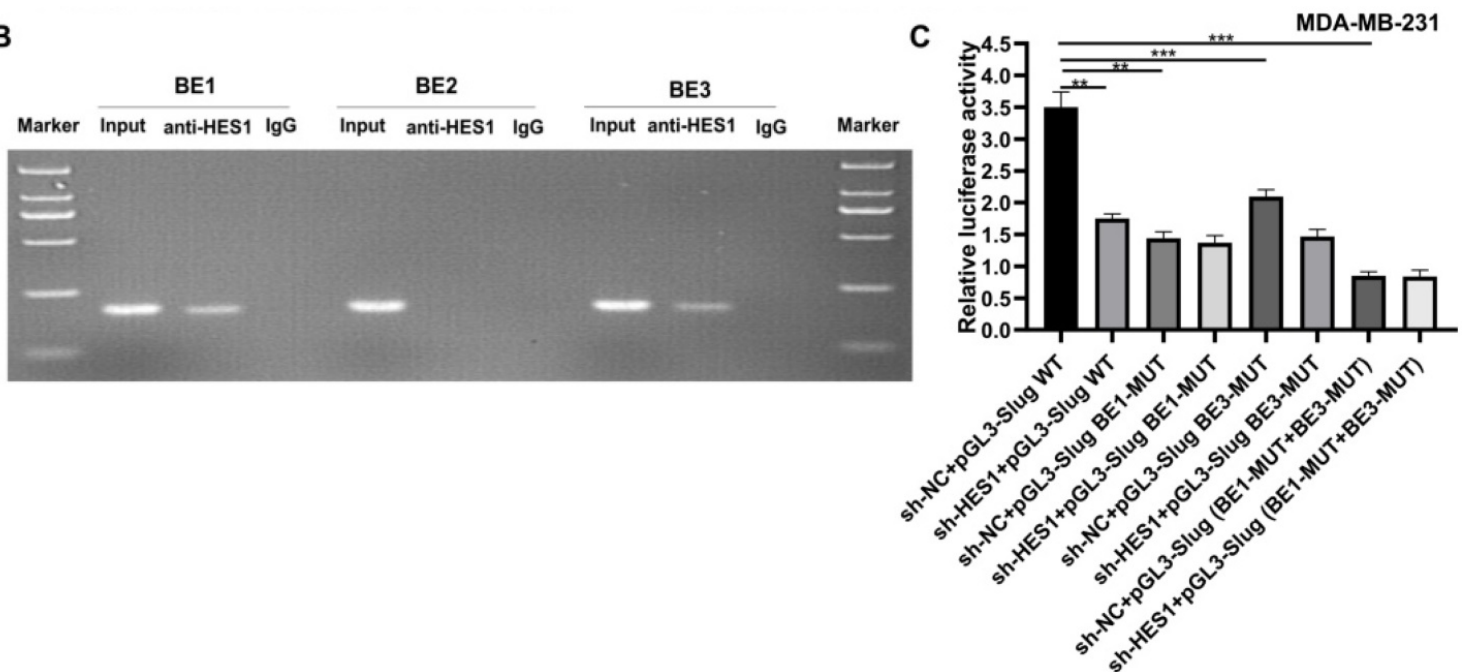

Figure 4. HESI activates Slug transcription. A) The schematic model showed the three putative binding elements (BEs) within Slug promoter region including BEl: 5'-AAACCCAGGTGCCTA-3' (-1644 to -1635); BE2: 5'-ATTTGCACGCGGCCGC-3' (-624 to -615); BE3: 5'-AGAGCGTGGA-3' (-241 to -232). B) HES1-binding sites within Slug promoter was detected in MDA-MB-23I cells by ChIP assay. C) Dual luciferase reporter assay in HESI-knockdown and control MDA-MB-231 cells, with co-transfection of wild-type or mutant-type Slug locus including BE1-MUT, BE1-MUT and BE1-MUT+BE3-MUT. Data are presented as the mean \pm SD of three independent experiments performed in triplicate. $* P<0.05, * * P<0.01, * * * P<0.001, * * * * P<0.0001$.

\section{HESI promotes Slug transcription}

To investigate whether HES1 functions as a transcriptional activator in regulating Slug expression, we analyzed an approximately 2000bp sequence upstream from the transcriptional start site of Slug gene by bioinformatics analysis. Bioinformatics prediction found that Slug had three putative HES1 binding elements (BEs) including BE1: 5'-AAACCCAGGTGCCTA-3' (-1644 to -1635); BE2: 5'-ATTTGCACGCGGCCGC-3' (-624 to -615); BE3: 5'-AGAGCGTGGA-3' (-241 to -232) (Figure 4A). In order to validate whether HES1 act directly on Slug promoter, we performed ChIP analysis on three putative BEs using HES1 antibody, with IgG as the negative control. The ChIP results revealed that the binding of anti-HES1 antibody was significantly enriched in BE1 and BE3, and there was no enrichment detected with BE2 and IgG control (Figure 4B). To further confirm HES1 binding enhanced Slug transcriptional activity, we conducted dual luciferase gene reporter assay in both MDA-MB-231 cells (sh-NC) and MDA-MB-231 cells with HES1 knockdown (sh-HES1) transfected with Slug promoter wild-type (Slug-WT) and Slug promoter mutant-type (BE1-MUT, BE3-MUT and BE1-MUT+ BE3-MUT) luciferase plasmids. The results showed that HES1 knockdown in MDA-MB-231 cells transfected with Slug promoter wild-type plasmid (sh-HES1/Slug-WT) decreased luciferase activity compared with the control group (sh-NC/Slug-WT). Whereas, there was no significant reduction of luciferase activity in HES1-knockdown cells transfected with Slug promoter mutant-type plasmids. Additionally, we also observed that MDAMB-231 cells transfected with Slug promoter mutanttype plasmids caused a strong decrease in luciferase activity compared with the control group (sh-NC/ Slug-WT) (Figure 4C). These results all demonstrated that HES1 promotes Slug gene transcription via binding to Slug promoter region.

\section{HESI contributes to BCSC stemness of TNBC through Slug}

To explore whether HES1 is a functional gene in BCSCs and how HES1 maintains stemness of BCSCs in TNBC, we investigated cancer stemness properties by a series of assays and their corresponding rescue assays. In TNBC cell lines (MDA-MB-231 and HCC-1937), the levels of stemness-related proteins such as SOX2, OCT4, Nanog were downregulated with loss of HES1, but restored when Slug was overexpressed upon HES1 knockdown (Figure 5A). HES1 knockdown remarkably reduced the capacity of 
tumorsphere formation and the ability of clonal formation and in TNBC cell lines (MDA-MB-231 and HCC-1937), but which were restored by upregulating Slug (Figure 5B-C). Based on well-recognized BCSC markers (CD44high CD24low) [21], BCSC populations (the ratio of $\mathrm{CD} 44^{\text {high }} \mathrm{CD} 24^{\text {low }}$ cell fractions) were clearly decreased upon HES1 knockdown in forming tumorspheres of TNBC cell lines (MDA-MB-231 and HCC-1937), whereas also restored by Slug overexpression. (Figure 5D). Thus, the above results all indicate that HES1 contributes to BCSC stemness properties and the impacts of HES1 on BCSC mediated by Slug in TNBC.

\section{HESI regulates BCSC stemness of TNBC through Slug in vivo}

To investigate whether and how HES1 influences BCSC stemness of TNBC in vivo, we examined tumor formation probability using mouse xenograft models. We constructed MDA-MB-231 tumorsphere cells mouse xenograft models. There are three groups: group 1: NC mice injected with MDA-MB-231/sh-NC tumorsphere cells; group 2: sh-HES1 mice injected with MDA-MB-231/sh-HES1 tumorsphere cells; group 3: sh-HES1+oe-Slug mice injected with MDA-MB-231/sh-HES1+oe-Slug tumorsphere cells. Consistent with in vitro results, transplantations of BCSC populations with limiting dilution assay revealed that the tumor formation probability was severely reduced upon HES1 knockdown and almost rescued by Slug overexpression (Figure 6A, Table 2). HES1 downregulation decreased BCSCs-derived tumor size and weight and the effects were restored by Slug overexpression in xenograft models with the limiting dilution of $1.0 \times 10^{5}$ cells per mouse (Figure 6B-D). The above data suggest that HES1 is closely related with tumor initiation both in vitro and in vivo. Taken together, our present results combined with our previous data verified that HES1 contributes to BCSC stemness of TNBC through Slug and HES1 upregulated Slug to activate EMT process and promote invasion in TNBC (Figure 6E).

Table 2. Statistical results of xenograft assay using tumorsphere cells upon HESI-knockdown and HES1-knockdown with Slug overexpression in TNBC cell line

\begin{tabular}{llll}
\hline Cell number & sh-NC & sh-HES1 & sh-HES1+oe-Slug \\
\hline 1000 cells & $2 / 6$ & $1 / 6$ & $2 / 6$ \\
10000 cells & $4 / 6$ & $2 / 6$ & $4 / 6$ \\
\hline
\end{tabular}

\section{Discussion}

In clinical practice, TNBC possesses the worst prognosis among all subtypes of breast cancers due to the lack of effective specific therapeutic targets. The reason for chemotherapy-resistance and recurrence of TNBC might be BCSCs enrichment in TNBC compared with other subtypes of breast cancers. Because large-scale current therapeutic strategies focus on eliminating the majority non-CSCs, the residual populations are recognized as chemo-resistance cancer cells, which derive from the existence of minority CSCs [22, 23]. Thus, seeking for effective therapies aiming at BCSC population of TNBC has attracted considerable attention worldwide. This led us to explore potential specific therapeutic targets for BCSC population of TNBC. Over the past few years, the close connection between CSC and epithelial to mesenchymal transition (EMT) has been demonstrated. The process of EMT not only enhances the migrative and invasive capacities of breast cancer cell, but also endows breast cancer cell with stemness properties [24]. Based on these, our previous reported study has demonstrated that HES1 expression is significantly high level in TNBC compared with other subtypes of breast cancer and HES1 leads to EMT process and promotes invasion. In our present study, to further investigate how HES1 regulates EMT, we explored the related mechanism by screening various well-known EMT-TFs including Twist, Snail, Slug, ZEB1, ZEB2 [14, 15], both in mRNA and in protein levels. In TNBC cell lines, most of EMT-TFs (Snail, ZEB1, ZEB2, Twist) were low endogenous level and showed mild or insignificant regulatory effects by HES1, but only Slug found to be high endogenously expressed and significantly consistent downregulation by HES1 knockdown. Our findings agree with others, which have demonstrated that Slug is endogenously overexpressed than other EMT-TFs in TNBC cell lines as well as TNBC patients and Slug is markedly higher in TNBC than that for other subtypes of breast cancer [25, 26]. The reason for that could be due in large part to the strong correlation between Slug and basal differentiation [25, 27] and also owing to the inverse correlation between Slug and ER expression in both breast cancer tissues and cell lines [28, 29]. Basal breast cancer cell almost is the negative for expression of ER, PR and HER2 negative in accordance with characteristics of TNBC by IHC [30, 31]. Moreover, Slug (Snai2), as a member of Snail family transcription factors, is well-known to promote cancer progression and cancer-associated EMT in a verity of cancers including breast cancer [32, 33]. In TNBC, Slug has been reported to be significantly associated with poor prognosis $[25,26$, 28 , 34]. The above all demonstrated the critical oncogenic role of Slug in breast cancer development. 
A

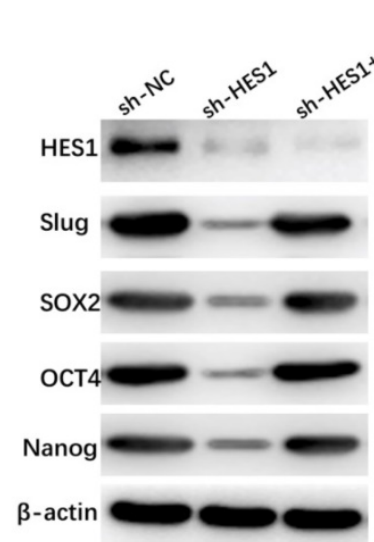

B
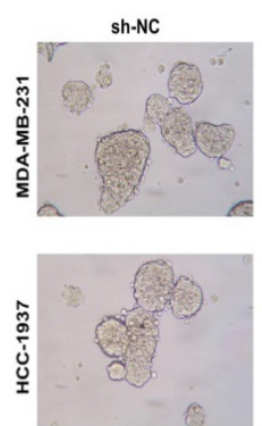

MDA-MB-231
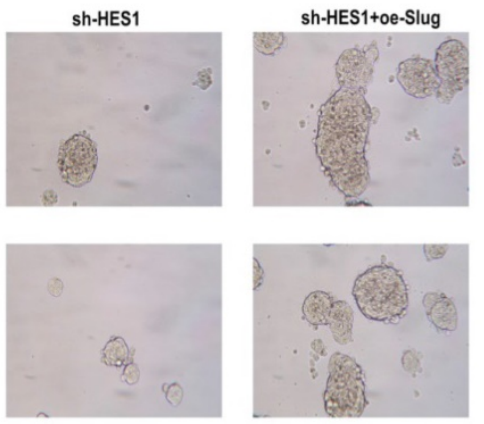

CC-1937
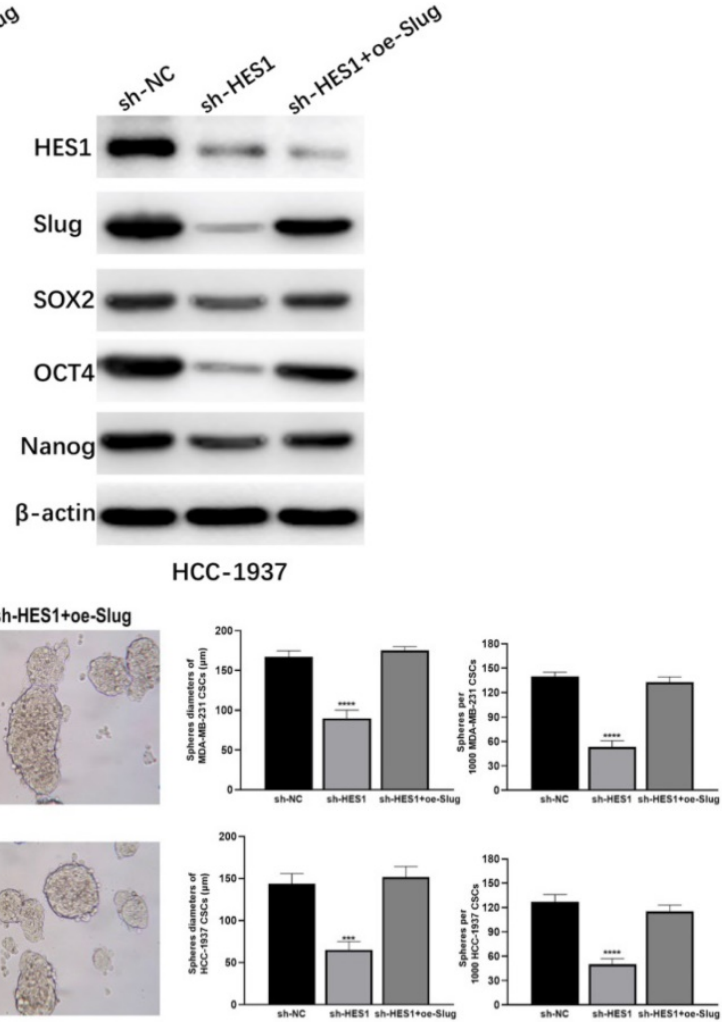

C
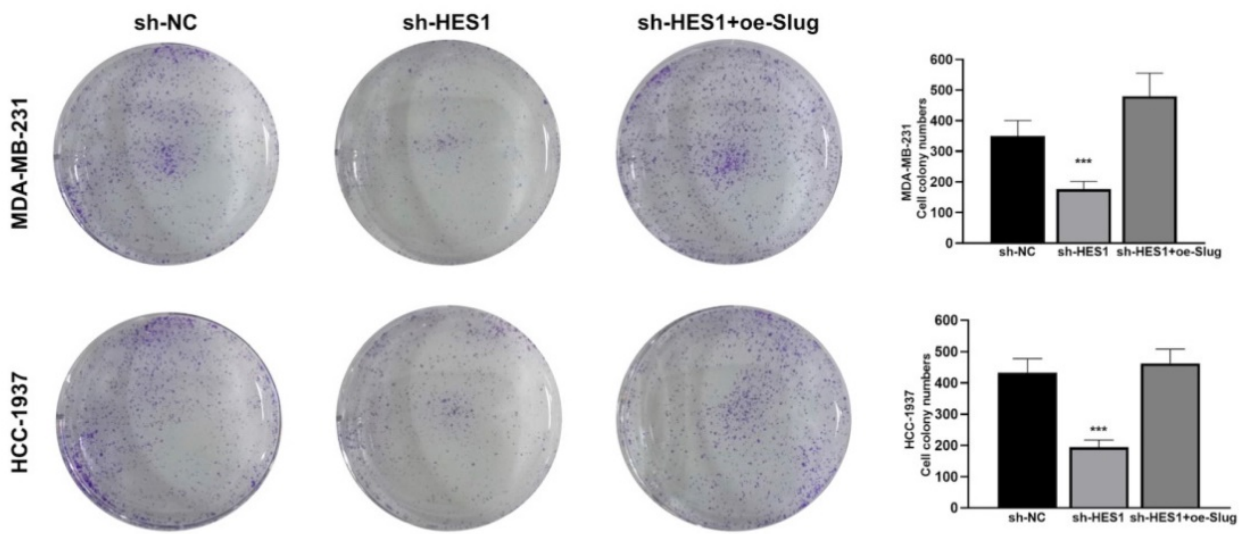

D
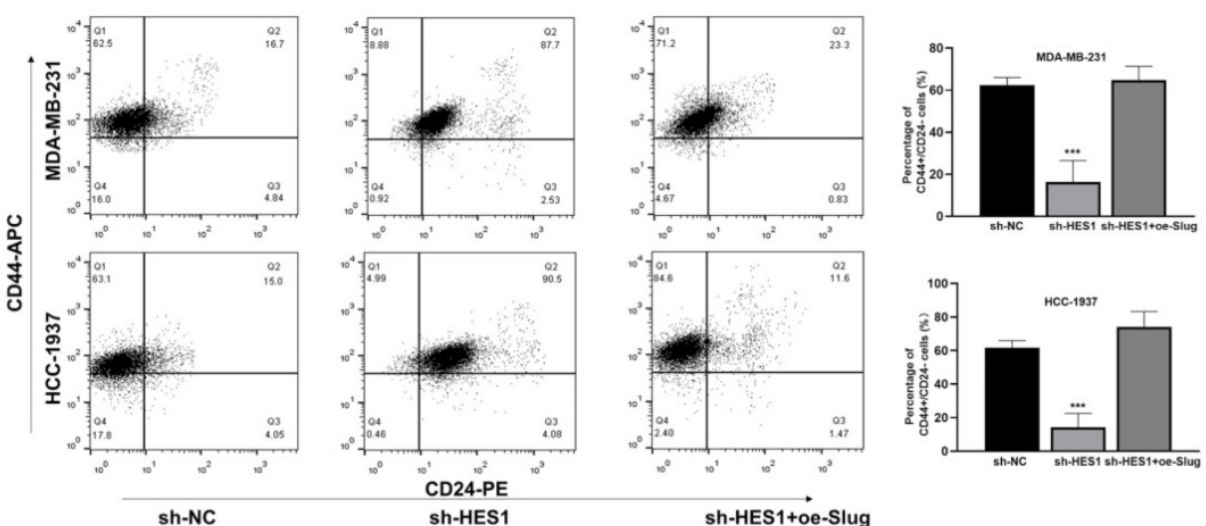

Figure 5. HES1 regulates BCSC stemness and cancer cell proliferation of TNBC through Slug. A) Protein levels of stemness-related genes (SOX2, OCT4 and Nanog) was detected in HES1-knockdown and HES1-knockdown with Slug-overexpression TNBC cell lines (MDA-MB-231 and HCC-1937). B) Tumorsphere assay upon HES1 knockdown and HES1 knockdown with Slug-overexpression in TNBC cell lines (MDA-MB-231 and HCC-1937). C) Clonal formation assay upon HES1 knockdown and HES1 knockdown with Slug-overexpression in TNBC cell lines (MDA-MB-231 and HCC-1937). D) Ratio of the CD44high CD24low populations was detected in tumorspheres of HES1-knockdown and HES1-knockdown with Slug-overexpression TNBC cell lines (MDA-MB-231 and HCC-1937) by flow cytometry analysis. Data are presented as the mean $\pm \mathrm{SD}$ of three independent experiments performed in triplicate. $* P<0.05, * * p<0.01$, $* * * p<0.001, * * * * p<0.0001$. 
A

$1.0 \times 10^{3}$ cells $\left(n=6\right.$ per group) $1.0 \times 10^{4}$ cells $(n=6$ per group $)$

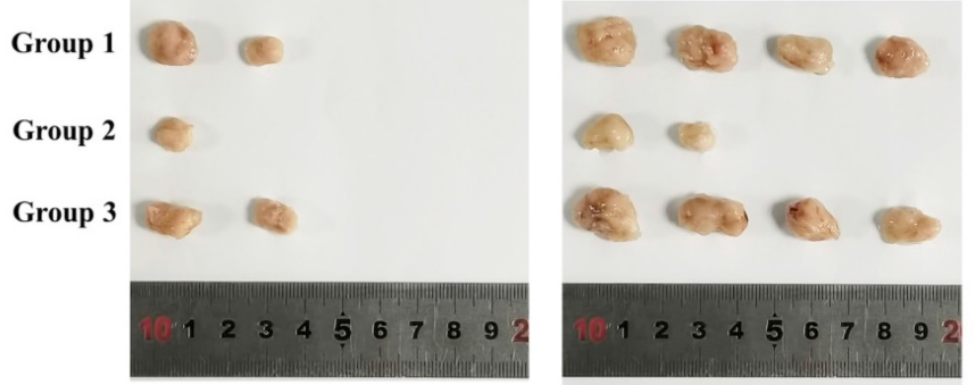

Group 1: sh-NC

Group 2: sh-HES1

B $1.0 \times 10^{5}$ cells ( $\mathrm{n}=3$ per group)

Group 3: sh-HES1+oe-Slug

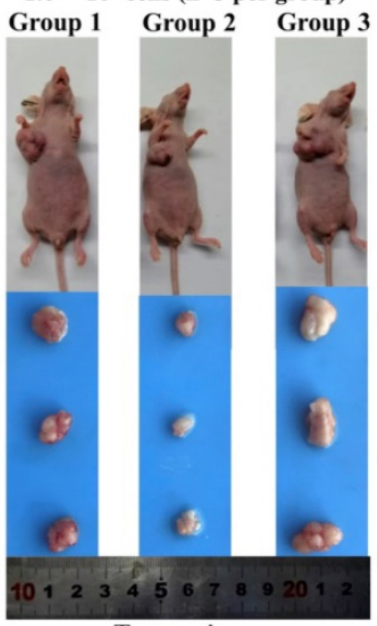

C
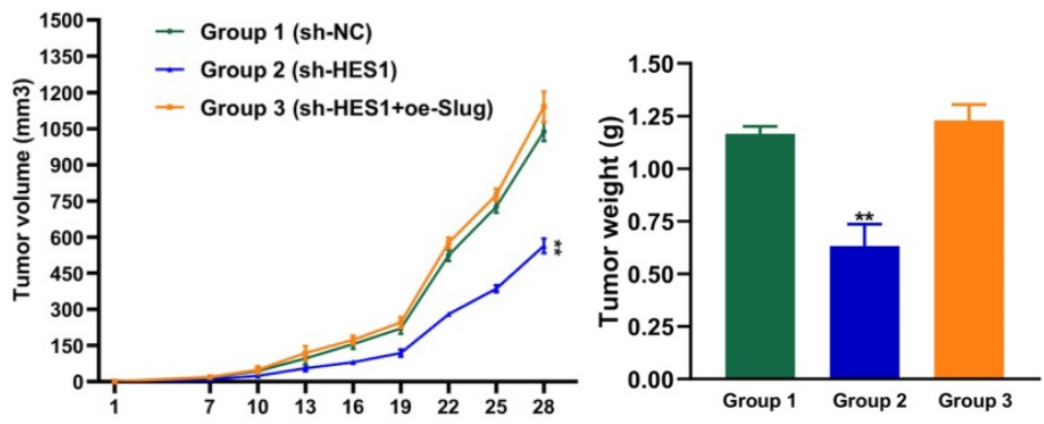

Group 1: sh-NC

Group 2: sh-HES1

Group 3: sh-HES1+oe-Slug

E

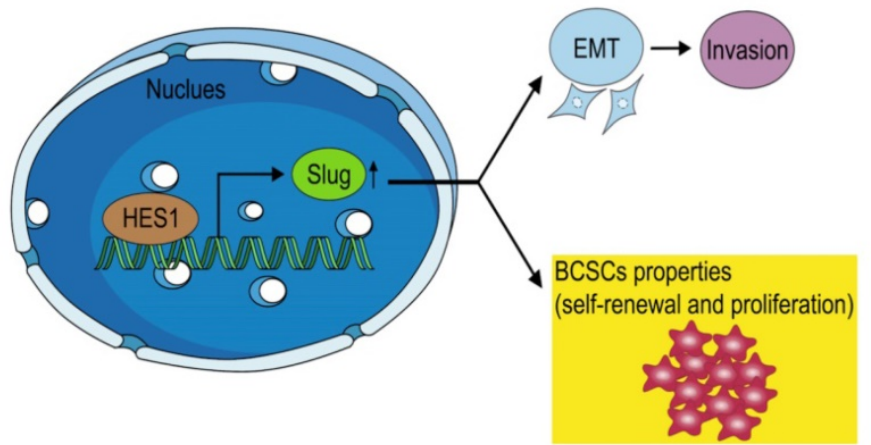

Figure 6. HESI regulates BCSC stemness of TNBC through Slug in vivo. A) Xenograft assay using tumorsphere cells $\left(1.0 \times 10^{3} ; 1.0 \times 10^{4}\right.$ cells per mouse, euthanized at seventh week) upon HESI knockdown and HESI knockdown with Slug-overexpression in MDA-MB-231 cell line. B) Xenograft assay using tumorsphere cells $(1.0 \times 105$ cells per mouse, euthanized at fifth week) upon HESI knockdown and HESI knockdown with Slug-overexpression in MDA-MB-231 cell line. C) Average tumor volumes of mice injected with $1.0 \times 10^{5}$ cells were measured in xenograft mice every three days. D) Average tumor weight of mice injected with $1.0 \times 10^{5}$ cells at the time of dissection. Data are presented as the mean $\pm S D$ of three independent experiments performed in triplicate. $* P<0.05, * * P<0.01, * * * P<0.001, * * * *<<0.0001$. E) The schematic model to show HES1 regulating BCSC stemness of TNBC through Slug as well as HESI inducing EMT and promoting invasion.

Thus, we focused on Slug as a critical specific trigger of EMT in TNBC. Meanwhile, we speculated that the remarkably downregulation of Slug by HES1-knockdown may be the main reason for affecting biological functions of TNBC. Subsequently, we revealed that there is a close correlation between HES1 and Slug expression in 150 TNBC patient specimens. Survival analyses indicated that HES1 leads to worse prognosis dependent on Slug. These results provide strong suggestions for that Slug may be a downstream gene of HES1. Moreover, many researches have revealed the critical role of Slug in promoting and maintaining self-renewal capacity of BCSC and inhibiting luminal differentiation. This is 
consistent with Slug's localization to the basal cell layer of the mammary epithelium, where mammary stem cells reside [25-27, 35, 36], whereas in luminal breast cancer, Snail or ZEB1 but not Slug is predominantly expressed and tightly associated with BCSC self-renewal capacities [25, 37]. This provides indications for EMT-driven BCSC stemness properties in different subtypes of breast cancer which is supposed to be triggered by diverse oncogenic signals. We therefore centered on Slug as a specific oncogenic factor responsible for EMT-driven stemness in TNBC, which implied that seeking the upstream regulator of Slug and blocking its regulation will be profoundly important in breast cancer research.

In addition, it has been reported that HES1 plays a critical role in blocking cell differentiation and promoting stemness properties in various cancers such as colon and pancreatic cancers [38, 39]. Moreover, several signaling pathways regulate HES1 such as canonical Notch and non-canonical Wnt, Hedgehog, MAPK and TGF- $\beta$ pathway $[30,41]$, which all are responsible for CSC stemness properties. Taken together, HES1 might serve as a core factor in the crosstalk of CSC-related signaling pathways [42, 43]. And HES1 may become a key biomarker and specific therapeutic target for BCSCs of TNBC. Thus, in our present study, data demonstrated that HES1 is a novel regulatory gene in BCSC self-renewal, BCSC population, cancer cell proliferation, and tumor formation of TNBC.

HES1 functions as a dual transcriptional factor and its role in transcription may depend on the HES1 transcriptional complex [9]. When HES1 interacts with a transcriptional co-activator, HES1 promotes its downstream gene transcription. As reported previously, HES1 has been involved in several cancer developments by its role in transcriptional activation or inhibition. In colorectal cancer, HES1 facilitated the aggressive progression via activating Bim1 transcription and repressing PTEN transcription [44]. HES1 promoted prostate cancer bone metastasis through an increasing in the transcriptional activity of RUNX2 [45, 46]. HES1 has been involved in promoting cancer cell transformation and increasing chemoresistance by an enhancement in the STAT3 transcriptional activity [47-49]. Considering that Slug is well known to highly correlated with BCSC self-renewal of TNBC [25-27, 35, 36], we speculated that the role of HES1 in regulating BCSCs of TNBC may be mediated by Slug. In our results of ChIP and dual luciferase gene reporter assays, these provided valuable evidences that HES1 is a novel transcriptional activator for Slug gene. Furthermore, the rescue assays from our in vitro data on the maintenance of BCSC stemness of TNBC as well as our in vivo data on the tumor formation of BCSC of TNBC, indicated that HES1 is involved in stemness of BCSCs in TNBC through Slug. Thus, our findings suggest that necessity of HES1 in the acquisition and maintenance of BCSC stemness of TNBC mediated by Slug.

Our study has some limitations. We selected MDA-MB-231 and HCC-1937 because these cell lines derived from triple-negative breast cancer and expressed endogenous high level of HES1 protein as well as Slug. More cell lines with other molecular subtypes can be used to further prove whether HES1 performs similar function. Although the method of BCSC induction and culture in this research has been approved by many previous reports, BCSCs identification and selection can be improved with more advanced technologies.

In conclusion, based on our previous as well as present findings, we have identified HES1 as a new BCSC maintaining protein for TNBC and found the regulatory network of HES1/Slug/EMT for its oncogenic role in promoting stemness properties in TNBC. Our studies highlighted a mechanism by which HES1 regulates BCSCs stemness properties and induces EMT process in TNBC via acting on Slug promoter region and activating its transcription. We propose that HES1 could serve as a crucial prognostic biomarker of TNBC and targeting HES1 might be a specific effective therapeutic strategy for TNBC in clinical practice.

\section{Acknowledgements}

This study was supported by grants from the National Natural Science Foundation of China (No. 81773163, 81702881) and China Postdoctoral Science Foundation (No. 2017M621178).

\section{Data availability}

All data supporting our findings can be found in the main paper, or made available on reasonable request from the corresponding author.

\section{Author Contributions}

Xiaoying Li, Feng Jin and Feng Li designed the study and conceived the project. Xiaoying Li, Yang Li, Xianqiang $\mathrm{Du}$ and Shu Guan performed the experiments and acquired the data. Xiaoying $\mathrm{Li}, \mathrm{Xu}$ Wang and $\mathrm{Yu}$ Cao analyzed the data. Xiaoying $\mathrm{Li}$ drafted the manuscript. Feng Jin and Feng Li revised the manuscript.

\section{Competing Interests}

The authors have declared that no competing interest exists. 


\section{References}

1. DeSantis CE, Ma J, Gaudet MM, et al. Breast cancer statistics, 2019. CA Cancer J Clin. 2019; 69: 438-51.

2. Bray F, Ferlay J, Soerjomataram I, et al. Global cancer statistics 2018: GLOBOCAN estimates of incidence and mortality worldwide for 36 cancers in 185 countries. CA Cancer J Clin. 2018; 68: 394-424.

3. Marotti JD, de Abreu FB, Wells WA, et al. Triple-Negative Breast Cancer: Next-Generation Sequencing for Target Identification. Am J Pathol. 2017; 187: 2133-8.

4. Bianchini G, Balko JM, Mayer IA, et al. Triple-negative breast cancer: challenges and opportunities of a heterogeneous disease. Nat Rev Clin Oncol. 2016; 13: 674-90.

5. Batlle E, Clevers H. Cancer stem cells revisited. Nat Med. 2017; 23: 1124-34.

6. Peitzsch C, Tyutyunnykova A, Pantel K, et al. Cancer stem cells: The root of tumor recurrence and metastases. Semin Cancer Biol. 2017; 44: 10-24.

7. Liu ZH, Dai XM, Du B. Hes1: a key role in stemness, metastasis and multidrug resistance. Cancer Biol Ther. 2015; 16: 353-9.

8. Goto N, Ueo T, Fukuda A, et al. Distinct Roles of HES1 in Normal Stem Cells and Tumor Stem-like Cells of the Intestine. Cancer Res. 2017; 77: 3442-54

9. Rani A, Greenlaw R, Smith RA, et al. HES1 in immunity and cancer. Cytokine Growth Factor Rev. 2016; 30: 113-7.

10. Hidalgo-Sastre A, Brodylo RL, Lubeseder-Martellato C, et al. Hes1 Controls Exocrine Cell Plasticity and Restricts Development of Pancreatic Ductal Adenocarcinoma in a Mouse Model. Am J Pathol. 2016; 186: 2934-44.

11. Sun L, Ke J, He Z, et al. HES1 Promotes Colorectal Cancer Cell Resistance To 5-Fu by Inducing Of EMT and ABC Transporter Proteins. J Cancer. 2017; 8: 2802-8.

12. Li X, Cao Y, Li M, et al. Upregulation of HES1 Promotes Cell Proliferation and Invasion in Breast Cancer as a Prognosis Marker and Therapy Target via the AKT Pathway and EMT Process. J Cancer. 2018; 9: 757-66.

13. Parvani JG, Schiemann WP. Sox4, EMT programs, and the metastatic progression of breast cancers: mastering the masters of EMT. Breast Cancer Res. 2013; 15: R72.

14. Peinado H, Olmeda D, Cano A. Snail, Zeb and bHLH factors in tumour progression: an alliance against the epithelial phenotype? Nat Rev Cancer. 2007; 7: 415-428.

15. Yang J, Mani SA, Donaher JL, et al. Twist, a master regulator of morphogenesis, plays an essential role in tumor metastasis. Cell. 2004; 117: 927-939.

16. Mani SA, Guo W, Liao MJ, et al. The epithelial-mesenchymal transition generates cells with properties of stem cells. Cell. 2008; 133: 704-15.

17. Creighton CJ, Chang JC, Rosen JM. Epithelial-mesenchymal transition (EMT) in tumor-initiating cells and its clinical implications in breast cancer. J Mammary Gland Biol Neoplasia. 2010; 15: 253-60.

18. Dontu G, Abdallah WM, Foley JM, et al. In vitro propagation and transcriptional profiling of human mammary stem/progenitor cells. Genes Dev. 2003; 17(10): 1253-70.

19. Shaw FL, Harrison H, Spence K, et al. A detailed mammosphere assay protocol for the quantification of breast stem cell activity. J Mammary Gland Biol Neoplasia. 2012; 17(2): 111-7.

20. Ponti D, Costa A, Zaffaroni N, et al. Isolation and in vitro propagation of tumorigenic breast cancer cells with stem/progenitor cell properties. Cancer Res. 2005; 65(13): 5506-11.

21. Muhammad Al-Hajj, Max S Wicha, Adalberto Benito-Hernandez, et al. Prospective identification of tumorigenic breast cancer cells. Proc Natl Acad Sci U S A. 2003; 100(7): 3983-8.

22. Creighton CJ, Li X, Landis M, et al. Residual breast cancers after conventional therapy display mesenchymal as well as tumor-initiating features. Proc Natl Acad Sci U S A. 2009; 106: 13820-5.

23. Dean M, Fojo T, Bates S. Tumour stem cells and drug resistance. Nat Rev Cancer. 2005; 5: 275-84.

24. Puisieux A, Brabletz T, Caramel J. Oncogenic roles of EMT-inducing transcription factors. Nat Cell Biol. 2014; 16: 488-94.

25. Ye X, Tam WL, Shibue $\mathrm{T}$, et al. Distinct EMT programs control normal mammary stem cells and tumour-initiating cells. Nature. 2015; 525: 256-60.

26. Guo W, Keckesova Z, Donaher JL, et al. Slug and Sox 9 cooperatively determine the mammary stem cell state. Cell. 2012; 148: 1015-28.

27. Phillips S, Kuperwasser C. SLUG: Critical regulator of epithelial cell identity in breast development and cancer. Cell Adh Migr. 2014; 8: 578-87.

28. J-W Bai, M-N Chen, X-L Wei, et al. The zinc-finger transcriptional factor Slug transcriptionally downregulates ERa by recruiting lysine-specific demethylase 1 in human breast cancer. Oncogenesis. 2017; 6(5): e330.

29. Ye $Y$, Xiao $Y$, Wang $W$, et al. ERalpha signaling through slug regulates E-cadherin and EMT. Oncogene. 2010; 29: 1451-62.

30. Patrycja Gazinska, Anita Grigoriadis, John P Brown, et al. Comparison of basal-like triple-negative breast cancer defined by morphology, immunohistochemistry and transcriptional profiles. Mod Pathol. 2013; 26(7): 955-66.

31. Maggie C U Cheang, Miguel Martin, Torsten O Nielsen, et al. Defining breast cancer intrinsic subtypes by quantitative receptor expression. Oncologist. 2015; 20(5): 474-82.

32. Catarina Castro Alves, Fatima Carneiro, Heinz Hoefler, et al. Role of the epithelial-mesenchymal transition regulator Slug in primary human cancers. Front Biosci (Landmark Ed). 2009; 14: 3035-50.
33. Hajra KM, Chen DY, Fearon ER. The SLUG zinc-finger protein represses E-cadherin in breast cancer. Cancer Res 2002; 62: 1613-18.

34. Wenyang Li, Minhong Shen, Yi-Zhou Jiang, et al. Deubiquitinase USP20 promotes breast cancer metastasis by stabilizing SNAI2. Genes Dev. 2020 Sep 17. doi: $10.1101 /$ gad.339804.120.

35. Bhat-Nakshatri P, Appaiah H, Ballas C, et al. SLUG/SNAI2 and tumor necrosis factor generate breast cells with CD44+/CD24- phenotype. BMC Cancer. 2010; 10: 411.

36. Proia TA, Keller PJ, Gupta PB, et al. Genetic predisposition directs breast cancer phenotype by dictating progenitor cell fate. Cell Stem Cell. 2011; 8: 149-63.

37. Chaffer CL, Marjanovic ND, Lee T, et al. Poised chromatin at the ZEB1 promoter enables breast cancer cell plasticity and enhances tumorigenicity. Cell. 2013; 154: 61-74.

38. Gao F, Zhang Y, Wang S, et al. Hes1 is involved in the self-renewal and tumourigenicity of stem-like cancer cells in colon cancer. Sci Rep. 2014; 4: 3963.

39. Abel EV, Kim EJ, Wu J, et al. The Notch pathway is important in maintaining the cancer stem cell population in pancreatic cancer. PLoS One. 2014; 9: e91983.

40. Ingram WJ, McCue KI, Tran TH, et al. Sonic Hedgehog regulates Hes1 through a novel mechanism that is independent of canonical Notch pathway signalling. Oncogene. 2008; 27: 1489-500.

41. Stockhausen MT, Sjolund J, Axelson H. Regulation of the Notch target gene Hes-1 by TGFalpha induced Ras/MAPK signaling in human neuroblastoma cells. Exp Cell Res. 2005; 310: 218-28.

42. Takebe N, Miele L, Harris PJ, et al. Targeting Notch, Hedgehog, and Wnt pathways in cancer stem cells: clinical update. Nat Rev Clin Oncol. 2015; 12: 445-64.

43. Amerizadeh F, Bahrami A, Khazaei M, et al. Current status and future prospects of transforming growth factor-beta as a potential prognostic and therapeutic target in the treatment of breast cancer. J Cell Biochem. 2019.

44. Gao F, Huang W, Zhang Y, et al. Hes1 promotes cell proliferation and migration by activating Bmi-1 and PTEN/Akt/GSK3beta pathway in human colon cancer. Oncotarget. 2015; 6: 38667-80.

45. McLarren KW, Lo R, Grbavec D, et al. The mammalian basic helix loop helix protein HES-1 binds to and modulates the transactivating function of the runt-related factor Cbfa1. J Biol Chem. 2000; 275: 530-8.

46. Zayzafoon M, Abdulkadir SA, McDonald JM. Notch signaling and ERK activation are important for the osteomimetic properties of prostate cancer bone metastatic cell lines. J Biol Chem. 2004; 279: 3662-70.

47. Marfels C, Hoehn M, Wagner E, et al. Characterization of in vivo chemoresistant human hepatocellular carcinoma cells with transendothelial differentiation capacities. BMC Cancer. 2013; 13: 176.

48. Gu F, Ma Y, Zhang Z, et al. Expression of Stat3 and Notch1 is associated with cisplatin resistance in head and neck squamous cell carcinoma. Oncol Rep. 2010; 23: 671-6.

49. Kamakura S, Oishi K, Yoshimatsu T, et al. Hes binding to STAT3 mediates crosstalk between Notch and JAK-STAT signalling. Nat Cell Biol. 2004; 6: 547-54. 